\title{
Neurobiology of schizophrenia and bipolar disorder Hans-Jórgen Mфller
}

\author{
Address: Psychiatric University Department Munich, Germany \\ from International Society on Brain and Behaviour: 3rd International Congress on Brain and Behaviour \\ Thessaloniki, Greece. 28 November - 2 December 2007 \\ Published: 17 April 2008 \\ Annals of General Psychiatry 2008, 7(Suppl I):S32 doi:I0.II86/I744-859X-7-SI-S32
}

This abstract is available from: http://www.annals-general-psychiatry.com/content/7/SI/S32

(C) 2008 Mфller; licensee BioMed Central Ltd.

An understanding of the neurobiology of schizophrenia and bipolar disorder has implications for treatment and outcome. The neurodevelopmental hypothesis of schizophrenia is of greatest importance in the neurobiological understanding of the aetiopathogenesis of schizophrenia. This hypothesis focuses on insults to prenatal brain development, which lead to brain alterations. Premorbid cognitive disturbances as well as behavioural abnormalities are interpreted as vulnerability markers in the context of this neurodevelopmental theory and are seen as a consequence of the premorbid brain alterations. Given the fact that heritability alone cannot explain schizophrenia or bipolar disorder, nongenetic factors impairing development must also be part of a multifactorial aetiopathogenesis of these disorders. The neurodevelopmental models have varied considerably with respect to specificity and timing of hypothesized genetic and environmental "hits".

In recent years longitudinal brain imaging studies of both early and adult onset populations with schizophrenia indicate that progressive brain changes are more dynamic than previously thought, with grey matter volume loss particularly striking in adolescence and appearing to be an exaggeration of the normal developmental pattern. This supports an extended time period of abnormal neurodevelopment in schizophrenia in addition to earlier "lesions". In schizophrenia, grey matter decreases have been detected in temporal lobes, hippocampus, frontal lobes, thalamus, amygdala and cingulate, and abnormalities in prefrontal white matter, corpus callosum and the Posterior superior temporal gyrus. It is of special interest that schizophrenia susceptibility genes and chromosomal abnormalities, particularly examined for early onset populations, are associated with premorbid neurodevelopmental abnormalities. Postmortem human brain and developmental animal studies document multiple and diverse effects of developmental genes (including schizo- phrenia susceptibility genes) at sequential stages of brain development. Increased specificity for the most relevant environmental risk factors such as exposure to prenatal infection, and their interaction with susceptibility genes and/or action through phase-specific altered gene expression, now both strengthen and modify the neurodevelopmental theory of schizophrenia.

Structural neuroimaging studies of bipolar disorder patients have found increased white matter hyperintensities in periventricular white matter, frontal lobes, and basal ganglia.

These abnormalities occur more commonly in bipolar disorders than in schizophrenia. Evidence suggests reduced grey matter in prefrontal brain regions and anterior thalamus, and volume changes have also been found in the amygdala and hippocampus. Multiple cases of schizophrenia, bipolar disorder and concurrent psychosis and mood disorder occur in some families. There is statistically significant evidence that bipolar disorder occurs at an increased rate in relatives of schizophrenic probands and in the relatives of bipolar probands. Susceptibility genes have been found, some specific to schizophrenia, some to bipolar disorder and yet others that influence susceptibility to schizoaffective disorder, schizophrenia and bipolar disorder.

In the past years, in addition to the neurodevelopmental disorder a neuroprogressive brain disorder has been under discussion to explain the decline especially in the poor outcome subgroup of schizophrenic patients. This presentation will discuss the available findings about similarities and distinctions in the neurobiology of schizophrenia and bipolar disorder. 